\title{
Ontology-based Fault Diagnosis and Maintenance Process Generation of Electromechanical System
}

\author{
Yuguo $\mathrm{Xu}^{\mathrm{a}, *}$, Shixin Zhang ${ }^{\mathrm{a}}$, Yong $\mathrm{Li}^{\mathrm{a}}$, and Jiawang Liu ${ }^{\mathrm{b}}$ \\ ${ }^{a}$ Department of Equipment Support and Remanufacture, Academy of Army Armored Force, Beijing, 100072, China \\ ${ }^{b}$ Unit 77562, Chinese People's Liberation Army, Shigatse, 857000, China
}

\begin{abstract}
In view of the complex fault diagnosis theory of complex equipment electromechanical systems and the difficulty of all maintenance process maintenance personnel, the ontology was introduced as a technical tool for knowledge modeling. Based on the analysis of fault diagnosis, maintenance process of knowledge structure and electromechanical systems, and use of protégé, the fault diagnosis and maintenance process of knowledge models of electromechanical systems was constructed. The faults diagnosis and auxiliary generation of the maintenance process for electromechanical systems were realized through FaCT++ reasoner to infer the constructed ontology. The model was verified, and the fault diagnosis and maintenance process knowledge could be integrated. Sharing and improving the efficiency of knowledge organization and maintenance technical support provide new technical means for effectively managing knowledge in the field of using electromechanical systems.
\end{abstract}

Keywords: electromechanical system; ontology; fault diagnosis; maintenance process generation

(Submitted on October 9, 2018; Revised on November 17, 2018; Accepted on December 16, 2018)

(C) 2019 Totem Publisher, Inc. All rights reserved.

\section{Introduction}

The intensive use of modern technology in the design and manufacture of weapons and equipment has greatly increased the knowledge content of equipment. How to effectively integrate and manage the knowledge of complex and heterogeneous equipment maintenance has become a bottleneck problem in the maintenance of equipment maintenance technology. To solve the problems of multiple sources and complicated and unsystematic data for equipment maintenance and protection data, it is necessary to conduct comprehensive analysis and research based on the generation and application of maintenance data (knowledge) to provide basic support for maintenance support informatization.

Ontology technology applied to fault diagnosis and maintenance process knowledge bases realizes the efficient reuse of domain knowledge due to its clear and standardized shared concept features. Scholars have conducted in-depth studies on this aspect. Yu developed an ontology-based fault diagnosis knowledge management system with the help of the fault tree generation algorithm [1]. Zhao tried to solve the problems of incomplete diagnostics by ontology and shared difficulties in the hobbing machine fault [2]. Wang proposed an appropriate ontology construction method for the problem of heterogeneous knowledge in the domain of numerical control machine tool fault diagnosis [3]. Peng designed fault knowledge ontology for the naval gun supply system based on the decision tree connection method and tested its consistency by using the Pellet inference engine [4]. The space-to-case space relationship was matched by an ontology mapping algorithm and achieved certain diagnostic effects [5].

Therefore, this paper starts from the actual needs of the existing maintenance support related business, analyzes the data requirements for equipment maintenance support, builds the data (knowledge) ontology model, and implements the maintenance support application based on the ontology model, which mainly includes:

(1) Combining the elements, data, information, and knowledge related to maintenance support from multiple

\footnotetext{
* Corresponding author.

E-mail address: mountren@126.com
} 
perspectives and dimensions (such as the work process of all participants involved in maintenance support and equipment maintenance support in equipment life cycle, etc.), analyzing all types of data related to equipment maintenance support business, and building an overall model of equipment maintenance support data.

(2) Applying the ontology modeling tool (protégé) to build the ontology model of equipment maintenance support data.

(3) Studying the application of maintenance-based knowledge based on ontology, taking the generation of equipment fault diagnosis or equipment maintenance process as an example, applying FaCT++ inference tools for analysis, and providing relevant support for maintenance support.

The organization of this paper is as follows: the second part introduces the ontology and its reasoning, the third part applies protégé to implement ontology modeling of the electromechanical system, the fourth part analyzes the fault diagnosis and maintenance process generation of the equipment electromechanical system based on ontology, and the fifth part gives specific cases.

\section{Ontology and Reasoning}

\subsection{Ontology}

Ontology is thought to have joined the computer field in the 1960s and has continued to develop. Ontology can be understood as a standardized description of the nature of things. Gruber first proposed in 1993 that "ontology is a clear specification of a conceptual model," a definition that has been recognized by many ontology research peers [6]. In 1995, Gruber made a clearer definition of ontology: "Ontology is an accurate description of conceptualization" [6]. In 1997, Borst proposed that "ontology is a formal specification of a shared conceptual model" [7] and focused on the sharing of ontology [8]. So far, however, there is no unified definition of ontology. The complete ontology currently recognized by many ontological researchers should include concepts, relationships, functions, axioms, and instances of five ontology elements [9]. Concepts refer to all things, such as equipment, maintenance, personnel, and equipment; relationships refer to the existence of two or more concepts, such as containment and affiliation; and functions refer to the ability to use mathematical expressions. The relationship between axioms represents an eternal, unchangeable truth; examples are often used to represent more specific events. Ontology can be expressed as the canonical description of the nature of objective things, that is, the concepts and relationships of highly abstract things. It is the description and expression of these abstract concepts and relationships [9]. The main advantages of ontological thinking are clear definition, clear boundaries, and the ability to represent conceptual relationships or describe specific areas of knowledge. Therefore, ontological ideas are being widely used in more and more fields.

A variety of ontology description languages can be roughly divided into two categories: Web-based ontology description language and predicate logic based ontology description language. With the development and progress of internet technology, the ontology description language based on Web has become the mainstream ontology description language. Web-based description languages include SHOE, XOL, OIL, RDFS, DAML+OIL, and OWL [10]. SHOE is an extension of HTML ontology; XOL is an ontology exchange language of XML; and OIL, RDFS, DAML +OIL, and OWL are all extensions of RDF that continue to inherit the RDF syntax and expressiveness. The currently popular ontology description languages include DAPL (DARPA Agent Markup Language), DL (Description Logic), RDF, RDFS (RDF Scheme), Ontolingua, OIL (Ontology Interchange Language), XOL (XML-Based Ontology Exchange Language), KIF (Knowledge Interchange Format), OWL (Ontology Web Language), OKBC (Opell Knowledge Base Connectivity), and Loom.

Ontology modeling methods include the skeleton method [11], TOVE method [12], seven-step method [13], IDEF5 method, METHODOLOGY method, KACTUS method, and SENSUS method [7]. At present, Apollo, WebOnto, WebODE, OntoEdit, and Protégé [14] are widely used and well-known modeling tools. Protégé uses a graphical interface that is easy for users to master and use, and it supports Chinese modeling. Protégé can implement editing and modification of ontologies in description languages such as RDF, OWL, and DAML+OIL. It is very suitable for the modification and description of maintenance knowledge.

\subsection{Ontology Reasoning Technology}

The main ontology reasoning techniques mainly include [15]:

(1) Based on description logic. The inference engines using this method are Pellet [16], Racer [8], and FaCT++ [17]. 
(2) Based on logic programming. The reasoning method implemented in the deductive database technology is the FOWL project. The KAON2 at the University of Karlsruhe in Germany is also a typical application of this technology.

(3) Based on first-order predicates. Use the first-order predicate proof mechanism to implement indirect reasoning of OWL statement statements converted to FOL. The ontology reasoning machine Hoolet [18] is the ontology reasoning realized by the Vampire first-order predicate prover.

(4) Based on rules. Since there are many mature OWL-to-rules conversion procedures, we can easily map ontology reasoning onto a rule inference engine. The results of inference can also be easily converted to OWL ontology for storage and sharing. Jess [19] and Jena are typical rule-based inference engines.

The logical reasoning basis of the ontology is Description Logic (DL). DL is also an ontology description language that unifies semantic networks, framework-based representations, object-oriented representation methods, and a logical basis for predicate logic, and it is often used to represent conceptual knowledge with a logical hierarchy [20]. The description logic system consists of four basic components: construction operator sets representing concepts and relationships, assertions of Tbox about conceptual terms, assertions of Abox about individuals, and logical reasoning mechanisms on Tbox and Abox. Tbox and Abox are used to assert two An instances in which individuals are related or used to assert a concept is an individual [21].

Protégé is an ontology modeling tool that is commonly used now. Its own inference engine FaCT++ is an inference based on description logic. Its structure principle is shown in Figure 1. Description logical reasoning generally includes Tbox (term set), Abox (assertion set), description logic language, and reasoning engine. The principle of description logical reasoning is to detect the knowledge of term sets and assertions, detect logical errors, and obtain implicit knowledge or information [20].

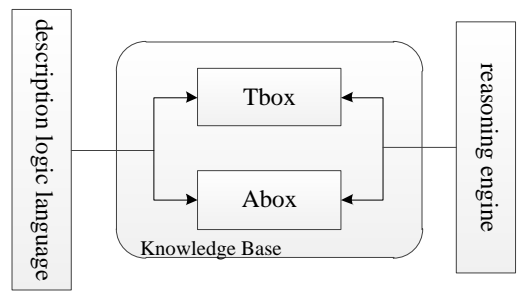

Figure 1. Schematic structure of FaCT++

\section{Equipment Electromechanical System Ontology}

\subsection{Requirement Analysis of Ontology in the Field of Equipment Maintenance Support}

In the several stages before the equipment is used and maintained, the data that may appear mainly include technical data, performance indicators, and various test data. The data sources mainly rely on the performance indicators and design data set by the R\&D institutions and the demonstration institutions, and the test institutions retain test data. In the use and maintenance phase of the equipment, the types of data that may be generated mainly include the motorized hours of the equipment and the fault and maintenance records of the equipment, and they mainly rely on the various resumes of the equipment to collect data. According to the idea of life-long maintenance, the equipment is divided into four categories: inservice equipment, equipment to be repaired, repair equipment, and decommissioning equipment. The data types of the equipment mainly include equipment number, repair unit, motorcycle hour, and maintenance record.

According to the idea of whole system maintenance, the participating organizations are divided into factories, use teams, management organs, repair institutions, scientific research institutions, training institutions, and equipment warehouse. The data types of participating organizations mainly include address, contact telephone, and organization structure. For demonstration personnel, office personnel, maintenance personnel, and equipment use personnel, the participants may include names, duties, specialties, ages, and technical grades. The maintenance and maintenance elements are divided into maintenance equipment, maintenance facilities, maintenance personnel, technical information, maintenance information, organizational structure, and dimension.

After the equipment is in service, the use of equipment, including the description of the use environment and usage, is mainly used to distinguish the influence of environmental factors such as different altitudes and different temperatures on the equipment and the impact of different usage methods on the equipment. The description of equipment maintenance includes various equipment maintenance resources, such as tools, equipment, facilities, funds, technical materials, and maintenance experience. 
Equipment maintenance support knowledge covers maintenance support personnel, maintenance equipment and facilities, maintenance technical information, maintenance experience, maintenance costs, maintenance instructions, and other factors. According to the characteristics of equipment maintenance support and the life cycle of equipment, maintenance support knowledge can be divided into equipment argumentation knowledge, development test knowledge, stereotyped production knowledge, use and maintenance knowledge, maintenance knowledge, and scrapped decommissioning knowledge. The knowledge of equipment use and equipment maintenance is of great significance for guiding equipment maintenance work, improving equipment design, accumulating maintenance support experience, and promoting improvement of maintenance support efficiency.

This paper focuses on the knowledge of equipment use and equipment maintenance and analyzes it from three aspects: fault knowledge description, maintenance support resources, and maintenance experience. Fault knowledge description is a comprehensive description of fault phenomena, fault locations, and fault causes, including all fault modes and fault types. Maintenance support resources are an important basis for completing equipment maintenance support tasks, including maintenance personnel, maintenance equipment, maintenance tools, equipment requirements, and technical data requirements. Maintenance experience includes equipment maintenance work regulations, various maintenance technical specifications, and expert experience formed in the actual work process.

\subsection{Characteristics of Equipment Maintenance Support Data}

Equipment maintenance support of equipment is a process of planning, organizing, coordinating, and controlling the coordinated operation of multiple departments with a scientific and reasonable plan, organization, coordination, and control. It mainly has the following characteristics:

(1) Complexity. Equipment failures caused by different use environments and intensity of use are often different. The maintenance support knowledge applied by different equipment and different levels of maintenance support organizations is often different. The knowledge of fault description and maintenance experience is also complex and diverse.

(2) Ambiguity. The complexity of maintenance experience and the diversity of fault description knowledge make certain knowledge vague and difficult to find a clear boundary.

(3) Relevance. The elements, data, knowledge, and information involved in the maintenance support process are interrelated and interdependent.

(4) Openness. Maintenance experience knowledge and fault knowledge are not static and have strong expansion capabilities.

\subsection{Modeling Steps of Equipment Maintenance Support Ontology}

The ontology modeling steps of equipment maintenance support involve determining the domain and category of knowledge ontology, checking the possibility of reusing existing knowledge ontology, listing important terminology in the ontology, and defining the hierarchy of classes, class attributes, attribute facets, and creating instances. The following example analyzes the modeling steps of failure and maintenance ontology on an equipment clutch.

(1) Determine the professional domain and category of knowledge ontology

Faults of clutches and their operating devices are mainly divided into two categories: one is where the clutch is not completely separated and the other is clutch slip. There are four reasons for both the incomplete separation and clutch slipping. Each cause is followed by a corresponding solution to the cause of the failure, which includes the same solution to the different causes and the same solution. The method corresponds to different causes of failure. The main clutch, operating device common faults, and exclusion methods are shown in Table 1.

(2) Check the possibility of reusing existing knowledge ontology

The Protégé [22] ontology library of Stanford University is well-known and allowed to be downloaded for free, and it can be comprehensively searched for data from multiple ontology databases. None of them can be reused in the field of equipment maintenance support. Therefore, by referring to the construction ideas of other domains ontology and combining the actual situation in the field of equipment maintenance and support, the most basic manual construction method is used for modeling. 
Table 1. Common clutch faults and troubleshooting

\begin{tabular}{|c|l|l|l|}
\hline No. & Fault phenomenon & \multicolumn{1}{|c|}{ Main reason } & \multicolumn{1}{|c|}{ Solution } \\
\hline \multirow{4}{*}{1} & \multirow{3}{*}{$\begin{array}{l}\text { Incomplete clutch } \\
\text { separation }\end{array}$} & Pipeline sealing & Add refueling and exhaust \\
\cline { 3 - 4 } & & Master cylinder push rod free stroke is too large & Adjust master cylinder push rod stroke \\
\cline { 3 - 4 } & & Friction plate warping & Replace friction plate \\
\cline { 3 - 4 } 2 & \multirow{4}{*}{ Clutch slip } & Control switch is damaged & Replace control switch \\
\cline { 3 - 4 } & & Friction plate with grease or excessive wear & Clean or replace friction plates \\
\cline { 3 - 4 } & & Compression spring is loose or damaged & Replace failed platen or spring \\
\cline { 3 - 4 } & & $\begin{array}{l}\text { Severe or severe wear of friction plates } \\
\text { not enough }\end{array}$ & Replace damaged friction plates or clutches \\
\hline
\end{tabular}

(3) List important terminology in the ontology

The common vocabulary of failure knowledge of a clutch and its control device mainly includes:

- Structure words: flywheel, connecting ring 1 (aluminum), connecting ring 2 (steel), intermediate pressure plate, clutch cover, compression spring.

- Fault name words: main clutch fault.

- Fault phenomenon words: slipping, not separating.

- Troubleshooting words: exhaust, replacement, cleaning, adjustment.

- Fault reason words: excessive free travel, wear.

- Maintenance method words: replacement pressure plate, cleaning friction plate.

(4) Define the hierarchy of classes and classes

The categories involved in this study include: equipment and equipment structure category, fault name category, fault cause category, fault phenomenon category, maintenance tools category, maintenance equipment category, maintenance personnel category, and maintenance method category.

(5) Define class attributes and attribute facets

The definition of class attributes and attribute partitions is to establish the relationship between the various classes, including relationship attributes and data attributes, wherein the relationship attribute is mainly used to describe the relationship between concepts and classes, and data attributes are used to describe the class or the relationship between objects and data types.

Definition of hasAbnormalPart (failure structure is): indicates the relationship between the equipment failure class and the equipment structure class.

The domain (Domin) of the attribute is the equipment failure class (FaultName), and the value domain is the equipment structure class (Fault structure).

Definition of hasPhenomenon (fault phenomenon is): indicates the relationship between the equipment failure class and the failure phenomenon class. The domain (Domin) of the attribute is FaultName, and the domain is FaultPhenomenon.

Definition of hasReason (the cause of the fault is): indicates the relationship between the equipment failure class and the failure cause class. The domain (Domin) of the attribute is FaultName, and the domain is FaultReason.

Definition of hasDisposal (solution is): the relationship between the failure cause class and the maintenance approach class. The domain of the attribute (Domin) is the FaultReason class, and the value domain is the FaultDisposal class.

At the same time, according to the actual situation of each class to establish the corresponding attribute, such as the definition of the attribute "equipmentCodeIs (equipment code is)" to represent the association between the equipment name class and the equipment code class, define the attribute "needToolIs (the required tool is)" to represent the relationship between the maintenance method class and the maintenance tool class. Define the attribute "personnelTechnicianGradeIs (personal technology level is)" to represent the association between the maintenance personnel name class and the maintenance personnel technical class. Define the data attribute "needToolNumberIs (tool requirements the quantity is )" to indicate the association between the maintenance method class and the data type "int". Because the tool requirement quantity can only be an integer, there is no decimal point, and all data types are "int". 
(6) Create an instance

\subsection{Realization of Fault Diagnosis Ontology of Electromechanical System}

Based on the analysis of electromechanical system faults and maintenance knowledge and the development process of the ontology, a core ontology model equipped with fault and maintenance knowledge for electromechanical systems is constructed using protégé. Taking clutch faults and maintenance as an example, there are six major categories of equipment, tools, fault causes, fault names, fault phenomena, fault locations, and maintenance personnel. Each major category is subdivided into corresponding subcategories. Until it can no longer be divided, the Viz structure of its class is shown in Figure 2.

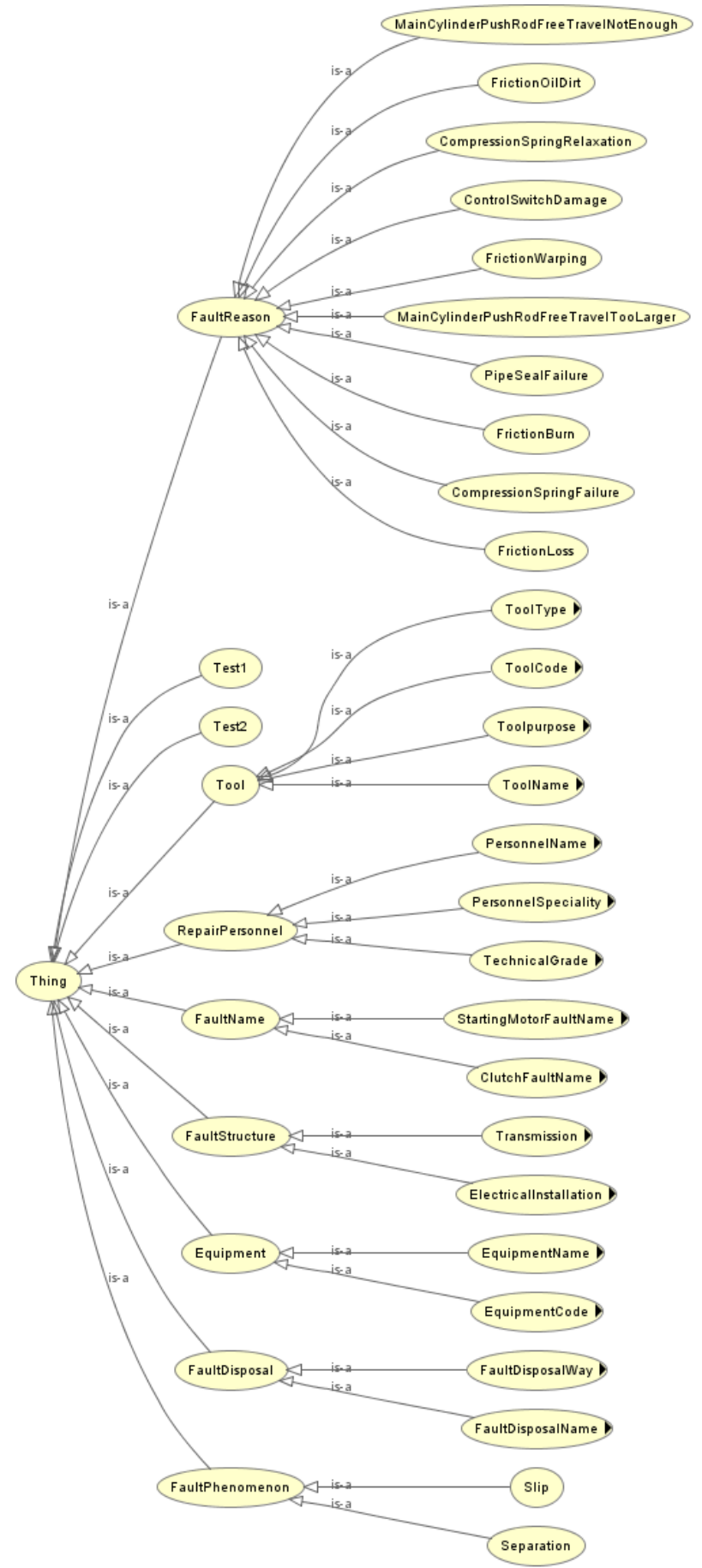

Figure 2. OWL Viz structure diagram 
At the same time, according to the characteristics of each object, the corresponding attribute is established to describe the relationship between the class and the class, including the relationship attribute and the data attribute. The relationship attribute mainly describes the relationship between the class and the class, and the data attribute is mainly described. The relationship is between classes and data types.

Figure 3 is a description of the attributes of the "Exchange Switch" for the maintenance method. The required attributes of the tools, personnel, and maintenance methods are described using the relationship attributes, and data attributes are used to describe the required number of tools and the number of personnel.

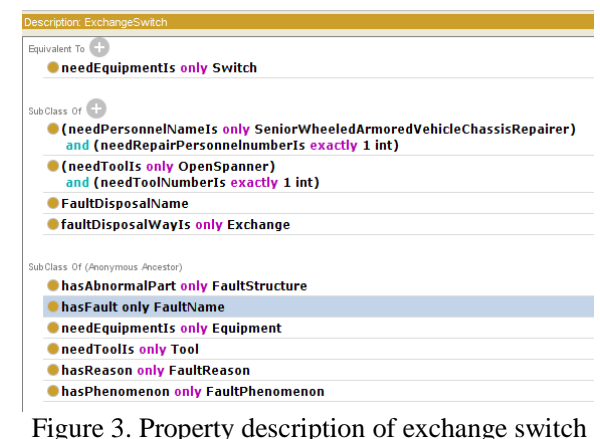

\section{Ontology-based Mechanical and Electrical System Fault Diagnosis and Maintenance Process Generation}

Protégé's own reasoning device FaCT++ is a reasoning based on description logic that can realize the reuse of maintenance support knowledge.

\subsection{Ontology-based Fault Diagnosis}

Use the description logic rules for reverse reasoning, such as the fault phenomenon to the cause of the fault and the location of the fault or the cause of the fault to the maintenance method. The rules are for complex types such as "fault name class" (compounded by the fault phenomenon class and the fault structure class). For equivalent description, the inference engine compares the attributes of the new class constructed with the attributes of the composite class to determine whether it is a subclass of the composite class. Create test class Test1 (fault diagnosis) and add the attribute "has Phenomenon only Slip" to test class Test1, as shown in Figure 4.

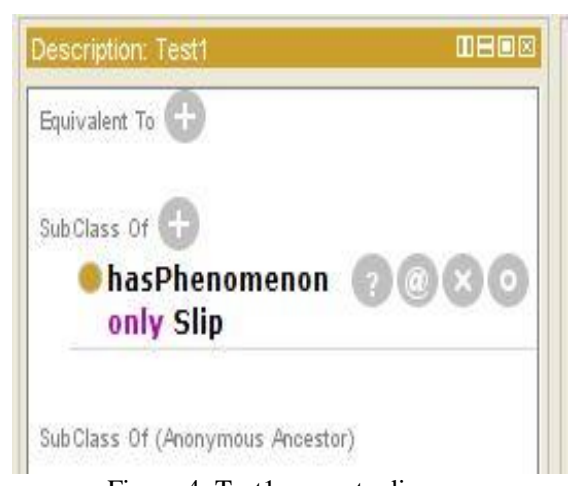

Figure 4. Test1 property diagram

The pre-inference class, Test1, is a subclass of the highest-level Thing class. After inference, Test1 becomes a subclass of "ClutchSlip" under "FaultName", as shown in Figure 5.

Class Test1 reasoning analysis: FaCT++ reasoning machine reasons out the properties of Test 1 class, as shown in Figure 6, pre-inference Test1 class adds only "has Phenomenon only Slip" after reasoning in addition to include "has Reasons why some Friction Burn", which may cause the clutch to slip. That is, "has Phenomenon only Slip", which includes "has Abnormal Part some Active Friction" and faulty parts, may have passive friction piece "has Abnormal part only Slip", and compression spring "has Abnormal part some Spring" is the description of the compression spring. The actual fault condition is consistent with the result of inference, that is, the reasoning result is correct. From the attribute of 
the inferred class Test1 (as shown in Figure 6), the ontology uses the description of the fault phenomenon to infer and then find the fault. The method of diagnosing the cause and the fault location is feasible.

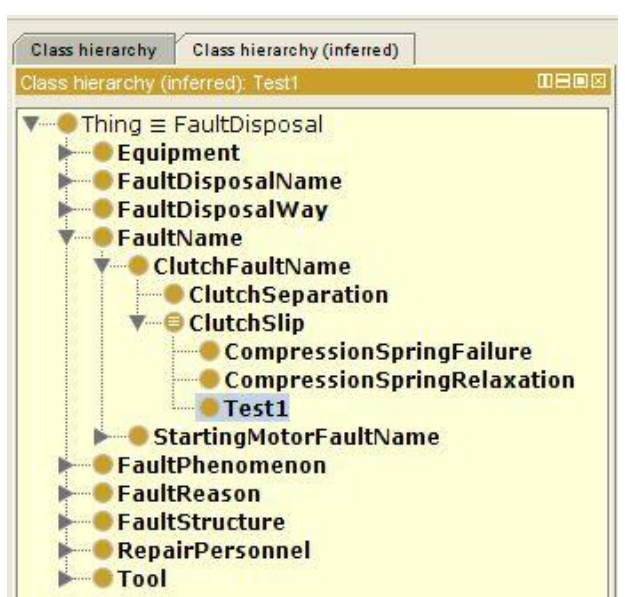

Figure 5. Class structure of Test1 after reasoning

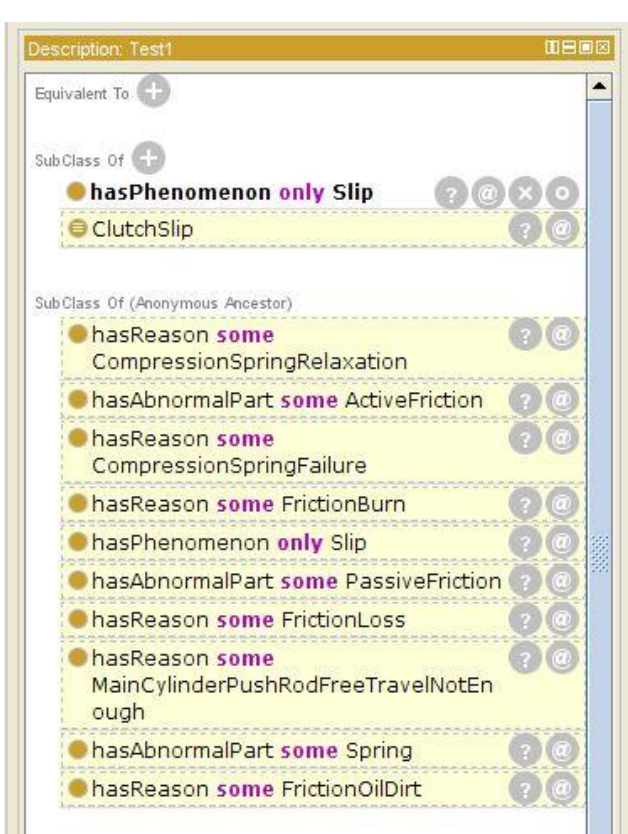

Figure 6. Class Test1 property diagram after inference

\subsection{Ontology-based Maintenance Process Generation}

A test class Test2 (maintenance process generation) is established, and a property "need Equipment only Switch" is added to the test class Test2, as shown in Figure 7.

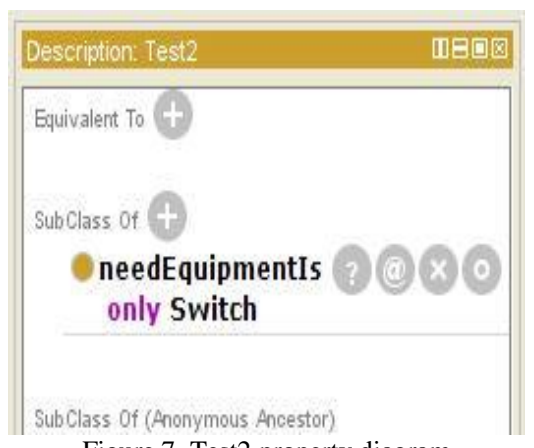

Figure 7 . Test2 property diagram 
After the reasoning, class Test2 becomes a subclass of "ExchangeSwitch (replacement control switch)" under "FaultDisposalName (maintenance method name class)", as shown in Figure 8.

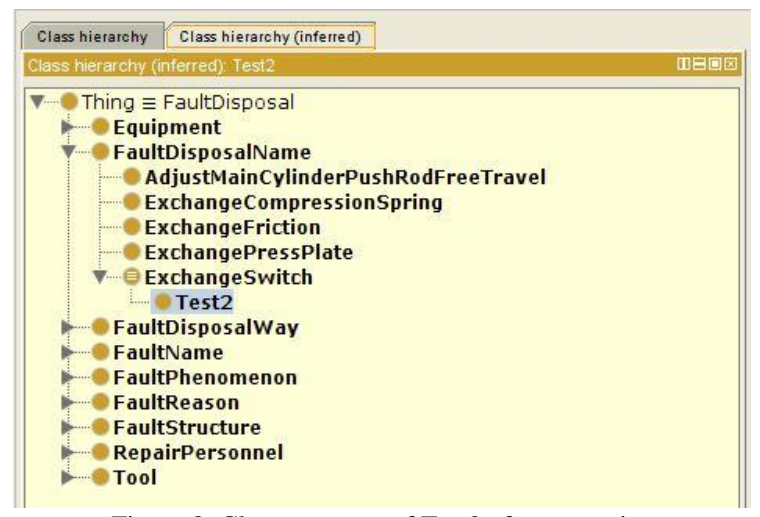

Figure 8. Class structure of Test2 after reasoning

The inference analysis of the test class Test2: the inferred attributes of the class Test2 are shown in Figure 9, including "fault Disposal Way Is Exchange", "need Personnel Name Is only Senior Vehicle Chassis Repairer and need Repair Personnel Number Is exactly 1 int", and "need Tool Is only Open Spanner and need Tool Number Is exactly 1 int". The maintenance demand situation is consistent with the result of inference, the reasoning result is correct, and the attribute of the inferred class Test 2 is used to explain the reasoning of the ontology using the description of the required equipment and then diagnose the maintenance personnel and the requirements of the maintenance tool. In the same way, reasoning from the cause of the fault to the repair method can be realized.

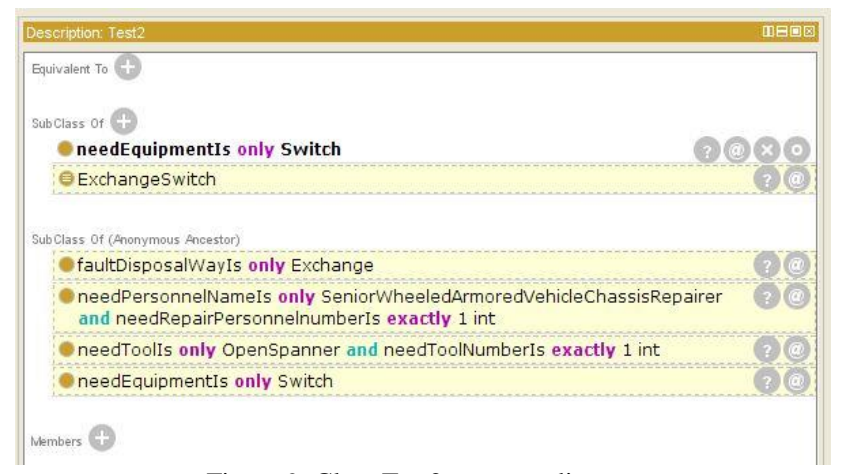

Figure 9. Class Test2 property diagram

\section{Conclusions}

In view of the complex fault diagnosis method and complicated theory of electromechanical systems, the difficulty of maintenance personnel, and the maintenance process, ontology and reasoning methods were introduced, and the ontology modeling tools were used to analyze the fault diagnosis and maintenance process knowledge structure of the electromechanical system. A knowledge model of fault diagnosis and repair process for electromechanical systems was constructed using protégé. The reasoning of the constructed ontology was implemented through the FaCT++ reasoner to realize the fault diagnosis of the electromechanical system and auxiliary generation of the maintenance process, and the model was verified. It provides new technical means for effective management of knowledge in the field of using electromechanical systems.

\section{References}

1. D. J. Yu, A. M. Zhou, and J. W. Guo, "Research on Fault Diagnosis Knowledge Management System based on Ontology," Journal of Hunan University (Natural Sciences), Vol. 38, No. 5, pp. 34-39, 2011

2. J. Zhao, P. Yan, and G. R. Chen, "The Research on Fault Diagnosis Knowledge Representation of Hobbing Machine based on Ontology," Manufacturing Automation, Vol. 33, No. 15, pp. 79-84, 2011

3. J. H. Wang, J. T. Huang, and B. Shen, "Research on Fault Diagnosis Ontology Modeling of CNC Machine Tools," Precision Manufacturing and Automation, No. 2, pp. 32-36, 2014

4. L. Peng, D. M. Yue, and C. G. Shi, "Research on Ontology-based Knowledge Modeling for Gun Feeding System Fault 
Diagnosis,” Ship Electronic Engineering, Vol. 34, No. 11, pp. 128-131, 2014

5. D. L. Qin, D. J. Yu, and J. Liu, "Ontology Driven Modeling of Diagnosis and Maintenance Knowledge for Mechanical Equipment," China Mechanical Engineering, Vol. 25, No. 14, pp. 1861-1866, 2014

6. R. Liffe, "Abstract Considerations: Disciplines and the Incoherence of Newton's Natural Philosophy," Studies in History and Philosophy of Science Part A, Vol. 35, No. 3, pp. 427-454, 2004

7. J. Li and L. S. Meng, "Comparison of Seven Approaches in Constructing Ontology," New Technology of Library and Information Service, No. 7, pp. 17-22, 2004

8. V. Haarslev and R. Moller, "Racer: A Core Inference Engine for the Semantic Web," in Proceedings of the 2nd International Semantic Web Conference (ISWC2003), pp. 292-297, Sanibel Island, Florida, 2003

9. L. Jiang and X. L. Zhang, "Review of Research about Ontology Concept," Journal of the China Society For Scientific and Technical Information, Vol. 26, No. 4, pp. 527-531, 2007

10. X. Y. Du, M. Li, and D. Z. Wang, "Survey on Semantic Web and Ontology," Computer Applications, Vol. 24, No. 10, pp. 1420, 2004

11. F. Yu, "Domain Ontology Construction Methods and Empirical Research," Wuhan University Press, Wuhan, China, 2015

12. Z. Q. Hu, "Ontology and Knowledge Organization," Chinese Literature and History Press, Beijing, China, 2013

13. N. F. Noy and D. L. McGuinness, "A Guide to Creating Your First Ontology," (https://protege.stanford.edu/publications/ ontology_development/ontology101.pdf)

14. X. M. Su and L. Ilebrekke, "A Comparative Study of Ontology Languages and Tools," in Proceedings of International Conference on Advanced Information Systems Engineering (CAISE), pp. 761-765, Toronto, Canada, May 2002

15. B. Xu, "Research on Health Management in Equipment Support based on Ontology," Jiangsu University of Science and Technology, Zhenjiang, China, 2012

16. E. Sirin, B. Parsia, and B. C. Grau, "Pellet: A Practical OWL-DL Reasoner," Journal of Web Semantics, Vol. 5, No. 2, pp. 5153, 2007

17. D. Tsarkov and I. Horrocks, "FaCT++ Description Logic Reasoner: System Description," in Proceedings of the International Joint Conference on Automated Reasoning, pp. 292-297, Seattle, 2006

18. D. Tsarkov, A. Riazanov, and S. Bechhofer, "Using Vampire to Reason with OWL," in Proceedings of the International Semantic Web Conference (ISWC2004), pp. 471-485, Hiroshima, Japan, 2004

19. E. J. Friedman-Hill, “Jess: The Rule Engine for the Java Platform,” (http://www.jessrules.com/)

20. D. Z. Xu, Z. Y. Wang, and B. Wang, "Comparison, Analysis and Research on Current Ontology Reasoners," New Technology of Library and Information Service, Vol. 1, No. 12, pp. 12-15, 2006

21. L. Shi and J. G. Sun, "Description Logic Survey," Computer Science, Vol. 33, No. 1, pp. 194-197, 2006

22. D. L. Rubin, N. F. Noy, and M. A. Musen, "Protégé: A Tool for Managing and using Terminology in Radiology Applications," Journal of Digital Imaging, Vol. 20, Suppl. 1, pp. 34-46, 2007 